Bibliotecas. Vol 35, №3, julio-diciembre, 2017. EISSN: 1659-3286

URL: http://www.revistas.una.ac.cr/index.php/bibliotecas/index

DOI: http://dx.doi.org/10.15359/rb.35-3.5

Licencia: Creative Commons (BY-NC-ND) 4.0 Internacional

\title{
Estudio de clima organizacional para los colaboradores de la "Biblioteca Joaquín García Monge de la Universidad Nacional, Costa Rica"
}

\section{Organizational Climate Study for the Collaborators of the "Biblioteca Joaquín García Monge of the Universidad Nacional, Costa Rica" \\ Máster Mildred Soto Vargas * \\ Universidad Nacional (UNA), Costa Rica}

\section{Resumen}

Toda organización, institución o empresa busca la excelencia, eficiencia y eficacia para alcanzar, de la mejor manera, las metas y objetivos propuestos en los tiempos establecidos. El papel que desempeña el recurso humano es fundamental para ejecutar las tareas de forma óptima, lo cual contribuye con el desarrollo de los procesos, para cumplir su misión y visión.

Reconocer que el recurso humano se encuentre motivado y comprometido; que la empresa o institución brinda una infraestructura apropiada; y que, además, se tome al colaborador en cuenta como parte fundamental de la institución, permitirá crear un clima organizacional adecuado.

Este trabajo de investigación está basado en la implementación de una consultoría de clima organizacional para los colaboradores de la Biblioteca Joaquín García Monge de la Universidad Nacional de Costa Rica, a través de la recolección de información por medio de la aplicación de un instrumento de recolección de datos (cuestionario). Con base en el resultado de la aplicación del instrumento se conocerán las variables que permiten medir las condiciones del clima laboral. Con el análisis de los resultados correspondientes se podrá detectar los diferentes factores que inciden en el clima organizacional del centro de trabajo, los cuales serán el insumo principal para realizar una propuesta de mejora, con el fin de obtener un mejor ambiente laboral. 
A nivel operativo la propuesta se centra en realizar un taller participativo a nivel de jefaturas, donde los ejes temáticos serán la comunicación y trabajo en equipo, evaluados por medio de dinámicas individuales y grupales. Al estar enfocado en las jefaturas del centro de trabajo, se tiene como expectativa que se replique en las diferentes secciones.

Palabras clave: biblioteca, clima organizacional, consultoría, comunicación, equipos de trabajo, recurso humano, talleres participativos.

\section{Abstract}

Every organization, institution or company seeks excellence, efficiency and effectiveness to achieve, in the best way, the goals and objectives proposed in the established times. The role played by the human resource is essential to perform the tasks optimally, which contributes to the development of the processes, to fulfill its mission and vision. Recognize that the human resource is motivated and committed; that the company or institution provides an appropriate infrastructure; and that, in addition, taking the collaborator into account as a fundamental part of the institution, will allow the creation of an adequate organizational climate.

This research work is based on the application of an organizational climate consultancy for the collaborators of the Joaquín García Monge Library of the National University of Costa Rica, done by the collection of information through the application of a data collection instrument (questionnaire). Based on the result of the application of the instrument, the variables that allow measuring the working environment conditions will be known. With the analysis of the corresponding results, it will be possible to detect the different factors that affect the organizational climate of the workplace, which will be the main input to make an improvement proposal, in order to obtain a better work environment.

At the operational level, the proposal focuses on conducting a participatory workshop at the headquarter level, where the thematic axes will be communication and teamwork, evaluated by means of individual and group 
dynamics. Being focused on the headquarters of the work center, it is expected that it be replicated in the different sections.

Keywords: library, organizational climate, consulting, communication, teamwork, human resources, participatory workshops.

\section{Introducción}

La consultoría se ha desarrollado como una actividad para medir o identificar un servicio, cuyos resultados permitan mejorar un servicio profesional de gran utilidad y ayudar a las altas gerencias a detectar los principales problemas o debilidades que afectan las organizaciones, instituciones o centro de trabajo. Asimismo, permite analizar las causas que provocan estos problemas planteando acciones para corregirlos y estableciendo un sistema de mejora continua. La labor del consultor como "agente de cambio" implica la transferencia de conocimientos y asesoría a los colaboradores de las organizaciones para que acepten nuevos retos e ideas y asuman riesgos.

La Universidad Nacional goza de gran prestigio a nivel nacional y posee gran renombre a nivel latinoamericano. Este prestigio la responsabiliza a utilizar, de manera adecuada, los recursos que se le asignan. Se hace imperante analizar a profundidad todas las variables del medio laboral, con el fin de determinar cuáles son las que más afectan el resultado de las labores diarias de los colaboradores.

Al conocer estas variables y estudiar sus injerencias en el recurso humano, las autoridades pueden determinar la forma de potenciar las fortalezas y minimizar las debilidades que existen en cada sección de este centro de trabajo.

Debido a lo anterior, el desarrollo de este proyecto con fines académicos, se centra en la realización de un estudio de clima organizacional en la Biblioteca Joaquín García Monge de la Universidad Nacional, para determinar los aspectos que están afectando el rendimiento de los colaboradores y así realizar una propuesta que permita hacer un cambio sobre las debilidades 
detectadas. Esta propuesta consiste en llevar a cabo un taller participativo que mejore el nivel de motivación de los colaboradores, el desarrollo del trabajo en equipo y una comunicación más asertiva que ofrezca mejores resultados en el rendimiento del recurso humano y que se traduce al final en un mejor servicio para los usuarios de la biblioteca.

La motivación del personal que labora en la biblioteca central de la Universidad Nacional va a reflejar un mejor servicio al usuario de la comunidad universitaria.

\section{Objetivos}

Los objetivos que se establecieron para efectos de este artículo son parte fundamental para el desarrollo del estudio de clima organizacional aplicado al personal de la Biblioteca Joaquín García Monge de la Universidad Nacional.

\section{Objetivo general}

Analizar el clima organizacional de la Biblioteca Joaquín García Monge de la Universidad Nacional para el segundo semestre del 2014 con el fin de realizar una propuesta de mejora.

\section{Objetivos específicos}

- Identificar los principales factores que intervienen en el clima organizacional de la Biblioteca Joaquín García Monge.

- Determinar las condiciones necesarias para el mantenimiento y/o mejoramiento del clima organizacional de la Biblioteca Joaquín García Monge.

- Implementar una estrategia para el mantenimiento y/o mejoramiento del clima organizacional para el personal de este centro de trabajo. 


\section{Antecedentes}

\section{a. Antecedente de la biblioteca Joaquín García Monge}

La Biblioteca Joaquín García Monge tiene su origen en la Escuela Normal de Costa Rica, de la cual heredó la infraestructura y el acervo bibliográfico de su biblioteca, especialmente orientado a la educación, debido a que la Escuela fue una institución dedicada a la formación docente. Posteriormente, en 1983 se inaugura el edificio donde actualmente se encuentra ubicada y dos años más tarde toma el nombre del distinguido benemérito de la patria y escritor nacional Joaquín García Monge.

La Biblioteca "Joaquín García Monge" es el nodo coordinador de El Sistema de Información Documental de la Universidad Nacional (SIDUNA), conformado por las Unidades de Información de Sedes Regionales y Facultades de la Universidad Nacional. Su misión y visión consisten en lo siguiente:

- Misión: brindar servicios y productos de información actualizados, eficaces y eficientes para contribuir al desarrollo de la docencia, investigación, extensión y producción académica de la Universidad Nacional de Costa Rica, trabajando con el soporte tecnológico, recursos humanos capacitados y calificados en forma coordinada e integral.

- Visión: disponer de un Sistema de Información Documental coordinado e integrado con canales de comunicación adecuados con los subsistemas en cada facultad, centro o sede. EI SIDUNA contribuirá con la visión institucional dirigida a prever los retos y desafíos actuales y futuros, con el fin de brindarle legitimidad y vigencia social, generando las posibilidades para el cambio y la innovación a la luz de los conocimientos y las necesidades emergentes del desarrollo futuro del país y mundial. 


\section{b. Descripción del área o procesos sujetos de estudio}

La Biblioteca Joaquín García Monge se encuentra ubicada en el Campus Omar Dengo de la Universidad Nacional, forma parte del Sistema de Información Documental de la Universidad Nacional. Su espacio físico cuenta con tres niveles de los cuales dos son de atención al usuario y el restante se encarga de los procesos administrativos. Este centro de información se divide en ocho secciones que se interrelacionen entre sí, como se describe en la Figura 1 sobre la estructura organizacional.

Las secciones que componente la biblioteca son:

- Dirección: coordina, planifica y supervisa las funciones de las diferentes secciones de la Biblioteca Joaquín García Monge y del SIDUNA con el fin de promover el desarrollo de los recursos bibliográficos. También brinda apoyo a las actividades de los programas de docencia, investigación, producción y extensión.

- Área Administrativa: realiza las actividades administrativas para el buen desempeño de las funciones de la Biblioteca Joaquín García Monge y del SIDUNA.

- Sección de Apoyo Informático: es la unidad encargada de dar soporte técnico a la Biblioteca Joaquín García Monge y a los centros de documentación que lo soliciten. Tiene a cargo la administración del software de sistemas de información documental ALEPH.

- Sección de Referencia y Educación al Usuario: es la sección encargada de infoalfabetizar al usuario en la búsqueda, recuperación y uso de los productos y servicios que se brindan en la Biblioteca Joaquín García Monge y en el SIDUNA.

- Sección de Circulación y Hemeroteca: facilita el préstamo de los documentos tanto a sala como a domicilio, como apoyo al proceso enseñanza-aprendizaje, compartiendo los recursos para el desarrollo de la docencia, investigación y extensión.

- Sección de Medios Audiovisuales: ofrece el servicio de préstamo de equipo, material audiovisual y salas equipadas como apoyo al quehacer académico institucional 
- Sección de Desarrollo de Colecciones: coordina el desarrollo del acervo documental, gestionando y registrando la adquisición de los requerimientos de información, solicitados por los programas de docencia, investigación, extensión y producción de la Universidad Nacional.

- Sección de Catalogación y Análisis Documental: es responsable de la catalogación, clasificación y análisis documental de los materiales en distintos soportes y formatos que ingresan al SIDUNA y otras dependencias universitarias.

Figura 1

\section{Estructura organizacional Biblioteca Joaquín García Monge}

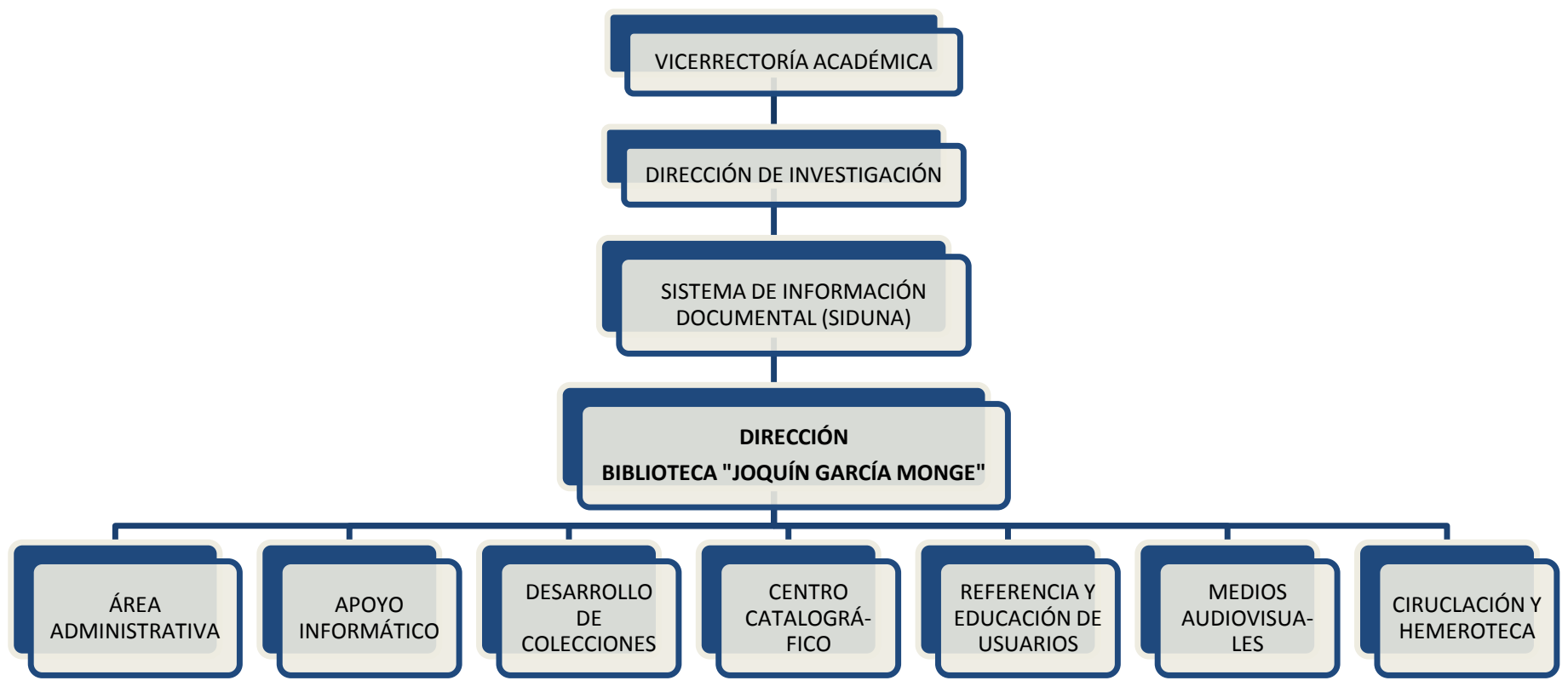

Fuente: C, Arrieta y M, Soto (2014), basado en información del sitio web www.siduna.una.ac.cr

\section{c. Alcances de la consultoría aplicada y delimitación}

- Alcances: conocer los factores, limitantes y aspectos generales vinculados al clima laboral de la Biblioteca Joaquín García Monge de la Universidad Nacional de Costa Rica para el segundo semestre del 2014. 
Se espera analiza los puntos de interés potenciales de la Administración sobre el mejoramiento del clima organizacional.

Con base a los resultados obtenidos, mediante los instrumentos aplicados a los colaboradores de la Biblioteca Joaquín García Monge, se llevará a cabo un taller a nivel de coordinadores de sección, con el cual se realizarán actividades que ayuden a mejorar los factores que se puntuaron bajos y que representan un factor de riesgo en la motivación de los funcionarios.

- Delimitación: el estudio se realiza en la Biblioteca Joaquín García Monge de la Universidad Nacional de Costa Rica.

- Población sujeta de estudio: la población de estudio está integrada por 60 colaboradores, ubicados específicamente en la Biblioteca Joaquín García Monge de la Universidad Nacional de Costa Rica. Debido a la cantidad de participantes no se requiere muestra de estudio; es decir, los instrumentos se aplicarán en la totalidad.

- Fuentes de información: la principal fuente de información se encontró en la entrevista a la directora de la Biblioteca Joaquín García Monge, Licenciada Margarita García Segura. A través de los instrumentos (cuestionarios, observación y entrevistas) aplicados a cada colaborador de la biblioteca, se logran medir diferentes factores del clima organizacional.

- Fuentes secundarias: se emplea el Reglamento de estructura y operacionalización del SIDUNA, consultado por medio del sitio web de la Biblioteca Joaquín García Monge: www.siduna.una.ac.cr

\section{Metodología}

En el nuevo enfoque administrativo se toman en cuenta varios aspectos trascendentales que inciden en el desarrollo y éxito de la empresa o instituciones, estos ejes determinan el buen funcionamiento de los procesos y actividades que se realizan. Es por esto que dentro del dinamismo estructural y jerárquico de la empresa se implementan nuevas actividades que permitan determinar las debilidades y fortalezas de cada uno de sus niveles, hasta llegar a un 
equilibrio adecuado para el buen funcionamiento de la empresa. El clima organizacional es uno de estos puntos angulares.

El centro de trabajo, objeto de esta investigación, pertenece a la Universidad Nacional, es la Biblioteca Joaquín García Monge, ubicado en el Campus Omar Dengo. Esta biblioteca cuenta con un edificio de tres niveles, el horario de funcionamiento es de lunes a viernes de 8:00 am a 9:30 pm y los sábados y feriados de 8:00 am a 5:00 pm. Este centro divide a su personal en tres horarios: el diurno, vespertino y horario especial.

La biblioteca central como nodo coordinador del Sistema de Información Documental (SIDUNA) busca innovar y mejorar la manera de realizar sus procesos de manera eficiente y eficaz a la hora de resolver las necesidades en temas de manejo de información.

La técnica utilizada en esta consultoría fue la entrevista, la observación y una encuesta tipo cuestionario. Para la recolección de datos del personal administrativo de la Biblioteca Joaquín García Monge se efectuó una entrevista a la Sra. Margarita García para conocer a nivel personal y profesional su criterio de cómo se encontraba el ambiente laboral del personal a su cargo. Así mismo, se aplicó una encuesta a los colaboradores tipo cuestionario con preguntas cerradas y abiertas, permitiendo tomar como referencia los resultados para ser evaluados y plantear una propuesta de mejora.

\section{a. Instrumentos utilizados para la recopilación de los datos}

Para efectos de este trabajo de investigación se utilizó un cuestionario diseñado para medir el clima organizacional del personal colaborador de la Biblioteca Joaquín García Monge de la Universidad Nacional de Costa Rica.

Este instrumento consistió en un conjunto de preguntas, cuyo objetivo es obtener información con algún objetivo concreto. Existen numerosos estilos y formatos de cuestionarios, de 
acuerdo con la finalidad específica de cada investigación; en este caso el cuestionario consta de cincuenta y ocho preguntas que se distribuyen en seis grandes temas:

1. Puesto de trabajo: se valoró el estado de ánimo del colaborador en el puesto que desempeña, las funciones que realiza y las metas impuestas.

2. Relación con los compañeros: se evaluó la relación del personal colaborador con los compañeros de trabajo.

3. Ambiente físico: se consideró la opinión del colaborador referente a las instalaciones de la biblioteca, cumplimiento de las normas de seguridad, condiciones de ruido, ventilación, luminosidad entre otros aspectos.

4. Estructura organizacional: se evaluó el sentir del colaborador referente.

\section{Análisis de la información}

En la primera parte de la consultoría se realizó la medición del clima organizacional de la biblioteca Joaquín García Monge para determinar los factores que está influyendo en la motivación de los colaboradores y que, al final, puede llegar a afectar el servicio para los usuarios de la biblioteca.

Entre los factores que se evaluaron, se detallan los datos generales por porcentajes. En el Cuadro 1 se visualiza la relación con los compañeros, el ambiente físico, el puesto de trabajo, el trabajo en equipo, la estructura organizacional y la comunicación. 


\section{Cuadro 1}

\section{Datos generales del estudio Clima Organizacional}

\begin{tabular}{|c|c|c|c|c|c|}
\hline \multirow{2}{*}{ FACTORES A EVALUAR } & $\begin{array}{c}\text { Siempre, } \\
\text { Muy Bueno }\end{array}$ & $\begin{array}{c}\text { Mayoría de } \\
\text { Veces, Bueno }\end{array}$ & $\begin{array}{c}\text { Pocas Veces, } \\
\text { Malo }\end{array}$ & $\begin{array}{c}\text { Nunca, } \\
\text { Muy Malo }\end{array}$ & $\begin{array}{c}\text { No Aplica, } \\
\text { No Utiliza }\end{array}$ \\
\hline \multirow{2}{*}{ Relación con los compañeros } & $55,09 \%$ & $41,20 \%$ & $2,78 \%$ & $0,46 \%$ & $0,46 \%$ \\
\cline { 2 - 6 } & Positivo & $\mathbf{9 6 , 3 0} \%$ & & Negativo & $\mathbf{3 , 7 0 \%}$ \\
\hline \multirow{2}{*}{ Ambiente físico } & $64,14 \%$ & $27,78 \%$ & $4,71 \%$ & $2,02 \%$ & $1,35 \%$ \\
\cline { 2 - 6 } & Positivo & $\mathbf{9 1 , 9 2} \%$ & & Negativo & $\mathbf{8 , 0 8} \%$ \\
\hline \multirow{2}{*}{ Puesto de trabajo } & $59,26 \%$ & $31,17 \%$ & $7,41 \%$ & $0,93 \%$ & $1,23 \%$ \\
\cline { 2 - 6 } & Positivo & $\mathbf{9 0 , 4 3} \%$ & & Negativo & $\mathbf{9 , 5 7 \%}$ \\
\hline \multirow{2}{*}{ Trabajo en equipo } & $47,41 \%$ & $41,11 \%$ & $8,89 \%$ & $1,11 \%$ & $1,48 \%$ \\
\cline { 2 - 6 } & Positivo & $\mathbf{8 8 , 5 2} \%$ & & $\mathbf{N e g a t i v o}$ & $\mathbf{1 1 , 4 8} \%$ \\
\hline \multirow{2}{*}{ Estructura organizacional } & $38,07 \%$ & $40,43 \%$ & $5,66 \%$ & $1,23 \%$ & $14,61 \%$ \\
\cline { 2 - 6 } & Positivo & $\mathbf{7 8 , 5 0 \%}$ & & $\mathbf{N e g a t i v o}$ & $\mathbf{2 1 , 5 0 \%}$ \\
\hline \multirow{2}{*}{ Comunicación } & $32,25 \%$ & $41,67 \%$ & $10,19 \%$ & $3,09 \%$ & $12,81 \%$ \\
\cline { 2 - 6 } & Positivo & $\mathbf{7 3 , 9 2} \%$ & & Negativo & $\mathbf{2 6 , 0 8} \%$ \\
\hline
\end{tabular}

Fuente: C. Arrieta y M. Soto (2014).

Por consiguiente, las preguntas abiertas del cuestionario que se le aplicó al personal colaborador de la Biblioteca Joaquín García Monge de la Universidad Nacional se describen en el Cuadro 2.

\section{Cuadro 2}

\section{Preguntas del cuestionario aplicado}

\begin{tabular}{|c|c|}
\hline Ámbitos & Respuestas de los encuestados \\
\hline \multirow{6}{*}{ 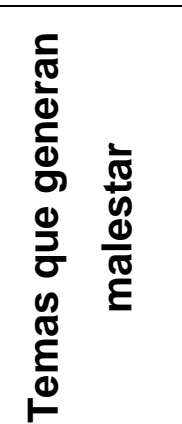 } & El jefe no motiva a los colaboradores $\left(20^{*}\right)$ \\
\hline & Poca posibilidad de crecimiento y desarrollo (13) \\
\hline & Los jefes los tratan mal (4) \\
\hline & No estar de acuerdo con la políticas de la biblioteca (4) \\
\hline & No le conviene el horario (4) \\
\hline & Salario insuficiente (4) \\
\hline
\end{tabular}


Bibliotecas. Vol 35, № 3, julio-diciembre, 2017. EISSN: 1659-3286

URL: http://www.revistas.una.ac.cr/index.php/bibliotecas/index

\begin{tabular}{|c|c|}
\hline & Existe acoso laboral (1) \\
\hline & Sin contestar (4) \\
\hline & Ambiente de trabajo agradable, buenas garantías, salario competitivo \\
\hline$\frac{\pi}{\omega}$ & Ambiente físico, estructura de la organización, relaciones personales \\
\hline & Beneficios laborales, cercanía geográfica y prestigio \\
\hline$\stackrel{0}{\frac{0}{2}}$ & Calidad humana y desarrollo de las funciones \\
\hline & Cantidad de beneficios para los funcionarios de la UNA \\
\hline ¿ & Cercanía a la casa \\
\hline & Estabilidad y horarios \\
\hline & $\begin{array}{l}\text { Mejor comunicación, más capacitaciones, fomentar trabajo en equipo y } \\
\text { más compromiso }\end{array}$ \\
\hline & No hay participación igualitaria \\
\hline$\frac{\pi}{0}$ & Las jefaturas no tienen liderazgo \\
\hline$\frac{\varepsilon}{\mathscr{J}}$ & Eliminar el correo de brujas \\
\hline$\stackrel{0}{2}$ & Mejorar las relaciones interpersonales \\
\hline \& & Falta de compañerismo con los otros departamentos \\
\hline d & Forma de pensar de algunas jefaturas. Servicio más personalizado \\
\hline 乐 & $\begin{array}{l}\text { Falta de capacitación, comunicación, toma de acciones en solución de } \\
\text { problemas }\end{array}$ \\
\hline & Reuniones mensuales para mejorar procesos de trabajo \\
\hline
\end{tabular}

* Número de veces que se indicó la respuesta.

Fuente: C Arrieta y M Soto (2014).

Como se muestra, la falta de comunicación vuelve a mencionarse como queja y el problema se agudiza cuando se observa que los colaboradores no se sienten motivados por las Jefaturas. Los colaboradores perciben que las Jefaturas no tienen liderazgo o que el trato hacia ellos no es el adecuado. 


\section{Propuesta para mejorar el clima organizacional de la Biblioteca Joaquín García Monge}

La propuesta es que mejore la comunicación y el trabajo en equipo, dirigida al personal colaborador de la Biblioteca Joaquín García Monge de la Universidad Nacional, con el fin de que se implemente a corto y mediano plazo por directriz de la directora de este centro de trabajo.

La propuesta se puede realizar en grupos de hasta 15 personas, pueden participar sin problema dejando al descubierto los servicios mínimos que se brindan a los usuarios. Específicamente esta actividad será aplicada en primera instancia a las jefaturas de las diferentes secciones que conforman la biblioteca central. Se escogió esta muestra de la población ya que estos colaboradores tienen una injerencia directa sobre el desarrollo del clima organizacional que impera sobre este centro de trabajo, además, trabajan con los mandos medios lo que da mayor objetividad para conseguir y llegar a los niveles operativos de la estructura que compone este centro de trabajo.

Se recomienda a la directora de la biblioteca que la actividad se desarrolle en un lugar ajeno al centro de trabajo, que cumpla con las condiciones apropiadas tanto en infraestructura como en logística, con el fin de crear un ambiente adecuado, el cual funcione como ventaja a la hora de implementar la actividad.

\section{a. Descripción y detalles de la propuesta}

Este trabajo toma como escenario la Biblioteca Joaquín García Monge de la Universidad Nacional, en el cual convergen profesionales que desempeñan una carrera común, pero que cada uno desempeña de forma tan independiente como las tareas que realizan. De igual forma existe bibliografía que se especializa en ciencias de la información donde se expresa cuáles son las necesidades y demandas que se ofrece para la atención de los usuarios en las bibliotecas. Asimismo, se ha escrito sobre las actitudes y formación que debe tener el nuevo 
profesional en sistemas de información documental, con el fin de que estos servicios sean con eficiencia y eficacia según las necesidades cambiantes que presenta el mundo moderno. Pero, ¿Qué se sabe del profesional bibliotecario? ¿Cómo cumplen sus tareas? De esos bibliotecarios que prestan sus servicios en nuestras bibliotecas ¿Cómo piensan? ¿Cuáles son sus necesidades, sus motivaciones y deseos? ¿Están motivados o no con su trabajo? ¿Consideran la biblioteca un buen lugar para trabajar? ¿En sus cargos hacen el mejor uso de sus habilidades?

La administración de algunas bibliotecas tiene como objetivo perfeccionarse en las áreas tecnológicas para dar un mejor tratamiento a la información. A la vez crean canales de comunicación con los clientes externos, como las redes sociales, buzones de sugerencias, con el fin de atender las necesidades planteadas, sin embargo, algunas veces no prestan la debida atención a quienes brindan el servicio o procesan la información.

Se puede dar el caso de que los profesionales en sistemas de información documental no se sientan valorados ni motivados socialmente, sin embargo, ellos representan la imagen de la biblioteca que cumple con una importante función para el público en general. A veces se olvida que para alcanzar la satisfacción de los clientes externos se debe lograr antes la satisfacción de los clientes internos. Sin embargo, no siempre se tiene interés en escuchar a nuestros colaboradores, conocer sus expectativas profesionales y personales, sus reclamos y sugerencias en relación con el trabajo que realizan, la integración entre los diferentes sectores de la institución u organización, la supervisión que reciben de sus superiores inmediatos y mediatos, la comunicación existente, su estabilidad, sus posibilidades de progreso profesional, los beneficios que le brinda la institución, el proceso de toma de decisiones y la mejor manera de trabajar en equipo, entre otros.

No se puede esperar el mejor resultado en los esfuerzos del personal colaborador si no se conoce nada de ellos, en cuanto a motivaciones o aspiraciones. Las organizaciones deben tomar las medidas necesarias para convertir a sus colaboradores en personas altamente efectivas y con resultados positivos. Conocer qué se puede necesitar, alcanzar la satisfacción 
laboral y hacer más atractivo su ambiente de trabajo, ayuda a brindar un mejor servicio a los usuarios. De esta manera, lo referencia la autora Alicia Delgado en su artículo de la Revista Interamericana de Bibliotecología, cuando hace mención de lo que plantea Lancaster (1994):

Aunque sea verdad que los desarrollos tecnológicos ponen algunas herramientas nuevas dentro de las bibliotecas y en manos de los bibliotecarios, estas herramientas no han sido necesariamente usadas de forma sabia o con imaginación en nuestra profesión. Pero... ¿por qué esa subutilización? se puede preguntar. Algunos podrían decir que se debe a una formación profesional ineficiente, pero... ¿no será el resultado de las dificultades de adaptación de ese profesional a un ambiente de trabajo o clima organizacional que no le ofrece las oportunidades que él desea?. (párr. 8)

En estos tiempos modernos ya existe una tendencia en el mundo institucional que busca una nueva concepción en los estilos de gestión, que tiene como foco principal el quehacer de las personas:

La vida de las personas se compone de una infinidad de interacciones con otros individuos y organizaciones. El ser humano es eminentemente social e interactivo; no vive aislado, sino en convivencia y en relación constante con sus semejantes. Por sus limitaciones individuales, los seres humanos se ven obligados a cooperar entre sí, a formar organizaciones para lograr ciertos objetivos que la acción individual y aislada no alcanzaría. (Chiavenato, 2009, p 6)

Por lo tanto, el tema del clima organizacional intenta medir, de alguna manera, factores como el ambiente, la estructura organizacional, la relación con los compañeros, la relación con la institución, entre otros. Sin embargo, de todos los enfoques el que ha demostrado mayor validez es el que utiliza, como elemento fundamental, las percepciones que las personas tienen de las estructuras y procesos presentes en su organización, empresa o institución. 
Esta propuesta pretende mostrar resultados obtenidos y analizados que arrojó la herramienta aplicada al personal colaborador de la Biblioteca Joaquín García Monge de la Universidad Nacional en el segundo semestre del año 2014, permitiendo medir factores como el puesto de trabajo, relación con los compañeros, ambiente físico, la relación con la institución, la estructura organizacional, la comunicación, el trabajo en equipo, entre otros.

Este instrumento fue elaborado con preguntas cerradas y abiertas para obtener mayor objetividad de los resultados. Según los datos obtenidos, se plantea desarrollar un taller que se enfoque en mejorar el clima organizacional en este centro de documentación e información, con el fin de potenciar las destrezas y aptitudes del personal colaborador, mantener y/o mejorar los canales de comunicación y trabajo en equipo para obtener una mejor satisfacción y motivación en el ambiente de trabajo.

\section{b. Objetivo general de la propuesta}

Realizar un taller de actividades para mejorar la comunicación y trabajo en equipo, dirigido al personal colaborador de la Biblioteca Joaquín García Monge.

\section{c. Objetivos específicos de la propuesta}

- Elaborar un plan de mejoramiento de los estilos de comunicación en la Biblioteca Joaquín García Monge.

- Promover el trabajo en equipo para el personal colaborador.

- Revisar la efectividad de comunicación a nivel de jefaturas con su personal colaborador.

- Implementar instrumentos de comunicación a lo interno de la Biblioteca Joaquín García Monge.

El fin de lograr dichos objetivos es mantener y mejorar el factor de comunicación y trabajo en equipo, se propone realizar un taller de actividades dirigido a grupos de 15 personas de 
distintas áreas de la Biblioteca Joaquín García Monge (directores, profesionales, técnicos, administrativos), los cuales constan de una jornada de cuatro horas y 30 minutos.

\section{d. Descripción y detalles del taller}

\section{Objetivo general del taller}

Aplicar herramientas que contribuyan al mejoramiento del clima organizacional en la Biblioteca Joaquín García Monge.

\section{Objetivos específicos del taller}

- Fomentar la comunicación, trabajo en equipo aumentando su capacidad de influir a nivel personal y laboral.

- Promover la reflexión personal y grupal de los participantes, incentivando la toma de conciencia de su propia acción personal como parte importante de la biblioteca.

Características: la naturaleza del taller es teórico - práctico, con metodología participativa, considerándolo totalmente dinámico a nivel personal y grupal, distribuido en jornadas de medio día de 4 horas y 30 minutos

Metodología: se aplicarán las técnicas orientadas al desarrollo de habilidades personales e interpersonales que hacen referencia a un conjunto de procedimientos racionales utilizados para alcanzar una gama de objetivos.

Metodología participativa: es un instrumento de primera línea para avanzar en el desarrollo de los profesionales en bibliotecología, considerándose una manera magnífica de oportunidad para mejorar la clase de personal colaborador en este centro de trabajo, donde se fomenten procesos de intercambio (experiencias, conocimientos...), de resolución colaborativa de problemas, de construcción colectiva de conocimientos, entre otros. 
Procedimiento: el taller se desarrollara en grupos de 10 a 15 personas.

\section{Contenidos}
- $\quad$ Comunicación
- $\quad$ Trabajo en equipo

Actividades propias del taller: la comunicación es el medio que nos permite una relación y un contacto con los demás. Algunas veces, la comunicación en los ambientes de trabajo no es sencilla, ni fácil de manejar, sin embargo, se pueden utilizar herramientas que permitan una mejor comunicación y, por lo tanto, genere un impacto de satisfacción en el personal colaborador.

En este taller se trabajará de manera integral, a través de la comunicación unos con otros, y se utilizarán los elementos que facilitan esta comunicación y se detectarán cuáles elementos la dificultan; así mismo, toma en cuenta el trabajo en equipo que es un pilar fundamental para cumplir los objetivos en las secciones de la Biblioteca Joaquín García Monge.

\section{Programa del taller}

Duración de las actividades: 4 horas.

- $\quad$ Bienvenida (5 minutos)

- Video de comunicación (20 minutos)

- Dinámica "Cuadros Rotos" (45 minutos)

- Dinámica, "Órdenes bien dadas" (30 minutos)

- $\quad$ Refrigerio (15 minutos)

- Comunicación con base en experiencias (90 minutos)

- $\quad$ Cierre (10 minutos) 


\section{e. Propuesta del taller aplicada a nivel de jefaturas de la Biblioteca Joaquín García Monge}

La propuesta del taller de actividades para mejorar la comunicación y el trabajo en equipo se llevó a cabo con la directora y las jefaturas de la biblioteca, en la sala de Proyecciones de la Sección de Medios Audiovisuales de la Biblioteca Joaquín García Monge de la Universidad Nacional. Se contó con el apoyo logístico y la alimentación por parte de la Sra. Margarita García, directora de la biblioteca. La idea de la implementación del taller a nivel de jefaturas, es crear un compromiso de replicar a sus colaboradores lo aprendido y ponerlo en práctica.

\section{Dinámicas a utilizar en el taller}

Las dinámicas desarrolladas en el taller fueron tomadas y adaptadas de gerza.com (es un sitio web que brinda actividades y orientaciones didácticas para trabajar con equipos diversos).

\section{Dinámica 1: "Cuadros rotos"}

\section{Objetivos:}

$>$ Analizar algunos aspectos de cooperación en la solución de problemas de grupo. $>\quad$ Sensibilizar a los participantes acerca de las conductas que obstaculizan o contribuyen a la solución de problemas de grupo.

Tiempo: Duración: 45 minutos

Tamaño del grupo: ilimitado. Divididos en subgrupos de 6 participantes.

Lugar: aula. Un lugar amplio, bien iluminado y acondicionado con una mesa y 6 sillas para cada subgrupo. 


\section{Material}

- Un juego de Cuadrados rotos para cada subgrupo.

- Hoja de instrucciones de "Cuadros Rotos" para cada subgrupo.

- Hoja de instrucciones del Juez / Observador de "Cuadros Rotos" para cada observador.

\section{Desarrollo}

El facilitador inicia con una plática sobre el significado de la palabra cooperación; esta plática deberá llevar a una hipótesis acerca de lo que es esencial para que el grupo coopere exitosamente en la solución de problemas. El facilitador indica que el grupo llevará a cabo un experimento para probar estas hipótesis. Es posible que surjan puntos semejantes a los siguientes:

1.- Cada uno en forma individual deberá comprender el problema en su totalidad.

2.- Cada uno en forma individual deberá comprender la forma en que se puede contribuir para la solución del problema.

3.- Cada uno en forma individual deberá estar consciente de la posibilidad de que otros participantes contribuyan.

4.- Existe la necesidad de reconocer los problemas de otras personas y ayudarlos para que realicen su máxima contribución.

5.- Los subgrupos que ponen atención en sus procesos de solución de problemas probablemente serán más efectivos, que los grupos que no lo hacen.

a) El facilitador forma subgrupos de cinco participantes más una persona que fungirá como el juez/observador. A estos observadores se les proporcionará una copia de la Hoja de Instrucciones para el Juez/observador. El facilitador pide a cada uno de los subgrupos que distribuyan entre sus miembros un juego de cuadrados rotos (dentro de cinco sobres). Los sobres van a permanecer cerrados hasta que se dé la señal de iniciar el juego.

b) El facilitador da a cada subgrupo una copia de las instrucciones, las lee y pregunta si hay dudas acerca de estas. 
c) Entonces se inicia el trabajo. Es importante que el facilitador visite todas las mesas durante el ejercicio para observar que se sigan las reglas establecidas en las instrucciones.

d) Cuando todos los subgrupos hayan terminado su tarea, el facilitador motiva a los subgrupos para que se discuta acerca del experimento. Se solicita que los jueces/observadores den sus observaciones. El facilitador alienta a los participantes para que relaten una experiencia de una situación real. En sesión plenaria el facilitador guía al grupo para que analicen cómo pueden aplicar lo aprendido en el campo laboral.

e) El facilitador guía un proceso para que el grupo analice cómo se puede aplicar lo aprendido en su vida cotidiana.

\section{Instrucciones para hacer el ejercicio de Cuadros rotos}

Un juego consiste en 5 sobres que contengan piezas de cartulina cortadas en diferentes formas, los cuales debidamente acomodados deberán formar 5 cuadrados del mismo tamaño. Un juego deberá ser entregado por cada grupo de 5 personas.
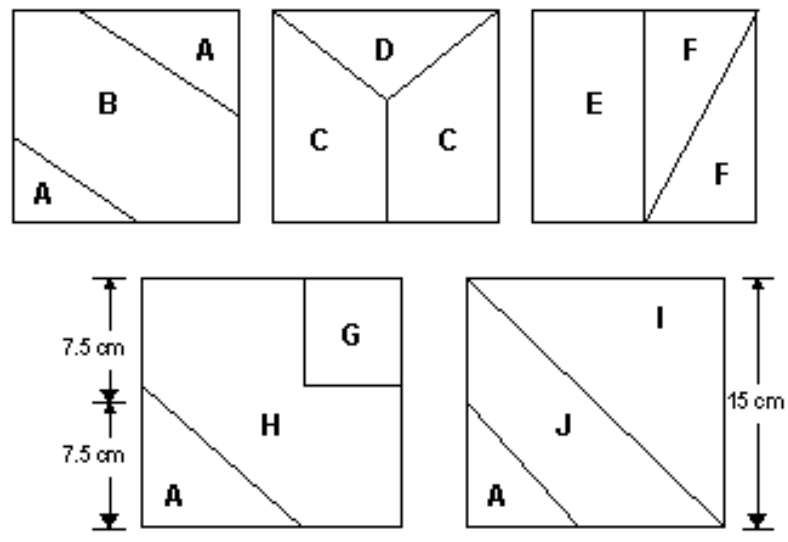

Para preparar un juego, se debe cortar cinco cuadrados de cartulina, cada uno debe medir exactamente $15 \times 15 \mathrm{~cm}$. Ponga los cuadrados en una fila y márquelos como se detalla, las letras deben ser marcadas ligeramente con lápiz para que posteriormente puedan ser borradas. Todas las piezas marcadas con la letra A, deberán ser del mismo tamaño, todas las que correspondan a la letra $C$ serán del mismo tamaño, y así sucesivamente. Es posible que 
diversas combinaciones formen uno o dos de los cuadrados, pero solamente una combinación formará los cinco cuadrados, cada uno de $15 \times 15 \mathrm{~cm}$. Después de trazar las líneas en los cuadrados y haber marcado las secciones con letras, corte cada cuadrado por las líneas señaladas para hacer las partes del rompecabezas.

Luego marque los cinco sobres de la siguiente forma: el sobre No. 1 contendrá: I, H, E; el 2 tendrá $A, A, A$, y $C$; el 3 tendrá $A$ y J; el 4 tendrá $D, F$ y el 5 tendrá $G, B, F$, y $C$. Borre las letras marcadas con lápices y en su lugar, escriba el número del sobre que contiene las piezas. Esto facilitará el guardar las piezas nuevamente en los sobres correspondientes. Cada juego deberá hacerse de cartulina de diferentes colores.

\section{Hoja de instrucciones para el grupo}

Cada uno de ustedes tiene un sobre el cual contiene piezas de cartulina para formar cuadrados. Cuando el facilitador dé la señal de empezar, la tarea de su grupo será la de formar cinco cuadrados de igual tamaño. La tarea no terminará hasta que cada uno de los participantes tenga delante de sí un cuadrado perfecto del mismo tamaño de los que se encuentran frente a los otros miembros del grupo.

Limitaciones específicas que se imponen al grupo durante la realización de este ejercicio:

1. No está permitido hablar.

2. No está permitido pedir a ningún miembro del grupo piezas, ni hacer señales, signos, entre otros, para solicitarlas. (Los miembros podrán voluntariamente darle piezas a los demás).

\section{Hoja de instrucciones del juez/observador}

Su misión es ser parte observador y parte juez. Como juez, deberá asegurarse de que se cumplan las siguientes reglas:

1. No se permite hablar, señalar o utilizar cualquier otro tipo de comunicación verbal y no verbal. 
2. Los participantes pueden dar piezas directamente a otros, pero no pueden tomar piezas de las otras personas.

3. Los participantes no pueden poner sus piezas en el centro para que los demás las tomen.

4. Se permite que cualquiera de los miembros dé todas sus piezas, aun cuando ya haya formado su cuadrado.

Como observador, ponga atención a lo siguiente:

1. ¿Quién está dispuesto a dar piezas de su rompecabezas?

2. ¿Hay alguno que cuando termina "su" rompecabezas se desentiende de los demás integrantes del grupo?

3. ¿Alguno de los participantes lucha con sus piezas, pero no es capaz de dar alguna o todas?

4. ¿Cuántas personas se encuentran comprometidas activamente en llevar a cabo su tarea?

5. ¿Cuál es el nivel de ansiedad o frustración?

6. ¿Hay algún punto en dónde el grupo empieza a cooperar?

¿Alguno trata de violar las reglas hablando o señalando para ayudar a alguno de los miembros a resolver sus problemas?

\section{Dinámica 2: “Órdenes bien dadas"}

Objetivo: evidenciar la manera en que damos las órdenes a nuestros colaboradores

Tiempo: duración: 30 Minutos

Tamaño del grupo: ilimitado

Lugar: aula

Material: fácil adquisición

- Dos Hojas Blancas.

- Dos lápices de grafito.

- Dos pupitres. 


\section{Desarrollo}

1. El facilitador solicita dos voluntarios. El resto del grupo observarán la dinámica.

2. Se le pide a cada voluntario que se siente uno de espaldas al otro en pupitres o mesas, cada uno con una hoja blanca y un papel.

3. El facilitador le pide al voluntario uno que libremente dibuje una casa.

4. Luego el facilitador le pedirá al voluntario uno que le vaya dando las indicaciones necesarias para que el voluntario dos copie el dibujo del voluntario uno, pero sin verlo. Deberán tratar de que quede lo más parecido posible.

5. Cuando terminen, comparen los dibujos.

6. Plenaria:

a) ¿Qué pasó? ¿Por qué los dibujos no quedaron iguales? ¿Se dieron las órdenes de manera correcta? ¿Se le pregunta al receptor si nos comprende?

Entre todos los participantes deben hacer una comparación de la dinámica con las situaciones que se presentan a diario en el trabajo.

\section{f. Producto entregado del taller a las jefaturas}

Se hace entrega de un manual de pasos del taller de comunicación y trabajo en equipo, con la descripción del taller, las actividades, el desarrollo de las dinámicas y el programa, en formato digital e impreso para que sirva de guía a las jefaturas para replicarlo a sus colaboradores.

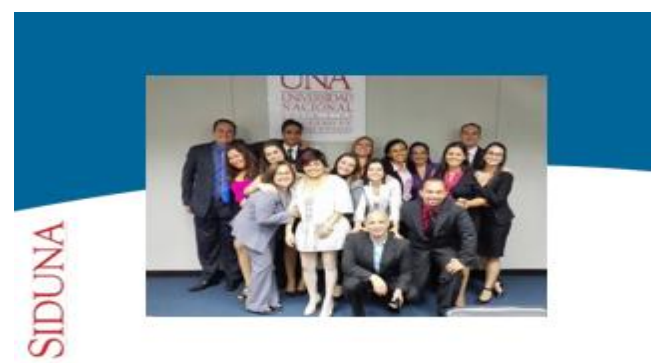

Taller de comunicación y trabajo en equipo
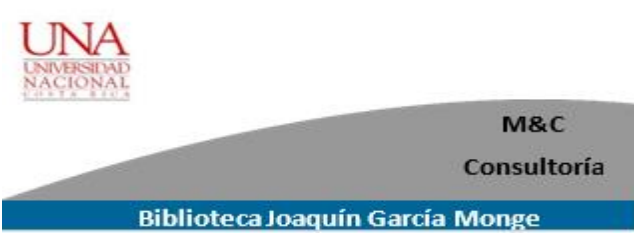

Fuente: Fotografía portada propias de la autora. 


\section{Conclusiones y recomendaciones}

- Se concluye que los factores que influyen en el clima organizacional en este centro de trabajo son los siguientes: ineficiencia en los canales de comunicación, así mismo, deficiencia en el trabajo en equipo, lo cual afecta directamente la relación Jefaturacolaborador y desemboca en desmotivación para el personal colaborador y un posible deterioro en los servicios que se brindan a los usuarios.

- Se debe establecer un plan de mejoramiento para fortalecer los canales de comunicación en la biblioteca tanto a nivel de jefatura como al resto del personal colaborador.

- Dentro de los requerimientos específicos para mantener y/o mejorar el clima organizacional está la implementación de líderes positivos en las jefaturas de la biblioteca y que estos prediquen con el ejemplo en el desarrollo de las secciones que tienen a cargo.

- En este centro de trabajo no existe, ni se ha intentado implementar, alguna estrategia para el mantenimiento y/o mejoramiento del nivel de comunicación del superior jerárquico con sus colaboradores.

- Se debe implementar la capacitación para desarrollar el liderazgo positivo en las jefaturas de las secciones, con lo cual se pueda actuar de manera adecuada en situaciones extremas, laborales y extra laborales, además de que se procure mantener al equipo de trabajo unido y motivado para que al mismo tiempo los servicios que brindan las secciones sean positivos y satisfactorios.

- Para este centro de trabajo se recomienda implementar como estrategia, un taller a nivel de jefaturas el cual contenga dentro de su desarrollo, herramientas que permitan potenciar las virtudes del personal al cual va dirigido y que pueda, al mismo tiempo, ser aplicado en el diario vivir y en el ámbito laboral.

- Como punto adicional se recomienda a la Biblioteca Joaquín García Monge aplicar el taller a todo el personal colaborador, ya que en esta ocasión se le aplicará a las jefaturas de este centro de información documental. Posteriormente, se recomienda a mediano plazo volver a aplicar el instrumento para controlar qué tan efectivo fue el taller para mejorar el clima organizacional. 


\section{Referencias}

Arrieta, C \& Soto, M. (2014). Estudio de clima organizacional para los colaboradores de la Biblioteca Joaquín García Monge de la Universidad Nacional de Costa Rica, Heredia, Costa Rica.

Chiavenato, I. (2009). Administración de Recursos Humanos. El capital humano de las organizaciones. 9nª ed. México: MacGraw-Hill.

Delgado Torres, Nora Alicia. (July/Dec 2006). Diagnóstico sobre las preferencias de clima organizacional de los bibliotecarios universitarios: el caso de las universidades de São Paulo y Antioquia. Revista Interamericana de Bibliotecología. 29(2). Recuperado se http://www.scielo.org.co/scielo.php?script=sci_arttext\&pid=S0120-09762006000200006

Gerza. (2012). Dinámicas de Grupo. Recuperado de: http://www.gerza.com/dinamicas/categorias/todas/desplegado_todas/c-d.html SIDUNA. Área de Soporte técnico de la Biblioteca Joaquín García Monge. (2014). Recuperado de: http://www.siduna.una.ac.cr/

\section{Notas de la autora}

* Máster Mildred Soto Vargas. Informática en la Biblioteca Joaquín García Monge (BJGM) y académica en la Escuela de Informática de la Universidad Nacional. Correo electrónico: mildred.soto.vargas@una.cr 